\title{
Optical Modeling of Photoluminescence of Multilayered Semiconductor Nanostructures: Nanowires, Nanotubes and Nanocables
}

\author{
Xue-Wen Chen and Sailing He \\ Centre for Optical and Electromagnetic Research, Zhejiang University; Joint Research \\ Centre of Photonics of the Royal Institute of Technology (Sweden) and Zhejiang University;
}

China

\section{Introduction}

One-dimensional semiconductor nanostructures, such as nanowires (NWs), core-shell nanocables (NCs) and nanotubes (NTs), have great potential in building up nanometric elements for photonic applications, such as light-emitting diodes (Yu et al., 1998; Könenkamp et al. 2004; Qian et al.,2005; Bao et al., 2006), nanolasers (Huang et al., 2001; Johnson et al.,2002; Duan et al.,2003), optical interconnects (Barrelet et al., 2004; Tong et al.,2005), biolabeling (Wang et al.,2005), sensing and spectroscopy. For such applications, light emission and guiding properties of the nanostructures are critical and determine the performance of the photonic devices. While the waveguiding and cavtiy properties of nanowires can be readily obtained by following conventional optical waveguide theory (Maslov E Ning,2003; Johnson et al.,2003), light emission properties of a nanostructure, due to the pronounced Purcell effect (Purcell, 1946; Lukosz, 1980), change in relatively subtle way as the dimension of the structure scales down. For example, as observed experimentally (Wang et al., 2001), photoluminescence (PL) of NWs shows a strong dependence on the polarization state of the excitation light, which cannot be fully explained by just taking account of the effect of excitation since spontaneous emission becomes highly dependent on the orientation of the transition dipole moment of the excitons as the size of the NW decreases (Chen et al., 2007a). Therefore, a rigorous and efficient optical model of light emission from one-dimensional semiconductor nanostructure is desirable for understanding the physics and for engineering the emission and its interaction with the nanostructure.

In this chapter, we present a comprehensive and efficient model for analyzing PL of a single multilayered nanostructure based on classical electrodynamics and, as concrete examples, investigate the emissions from a single $\mathrm{ZnO} \mathrm{NW}, \mathrm{NC}$ and NT as functions of geometrical parameters and the polarization of the excitation. Here we assume the excitation intensity for PL is well below the lasing threshold of the nanostructure and consequently spontaneous emission process dominates. Our theoretical model consists of two parts, namely, the modeling of the spontaneous emission of a single emitter in the structure and the evaluation of the excitation effect. The chapter is organized as follows. In section 2, we present a rigorous optical model of the spontaneous emission of an emitter in an arbitrarily multilayered structure and introduce an efficient numerical implementation of the model. 
Spontaneous emission properties, including the radiative decay rate, quantum efficiency and spatial distribution of the emission, are investigated for a single $\mathrm{ZnO} \mathrm{NW}$ and NT. In Section 3, a full optical model of PL is given by including the effect of the excitation. We show the strong dependences of PL intensity on the polarization of the excitation and the geometrical parameters of $\mathrm{ZnO}$-based nanostructures. Conclusion is given in Section 4 .

\section{Spontaneous emission in a cylindrically multilayered structure}

\subsection{Theory of spontaneous emission in nanostructures}

The theoretical description of spontaneous emission in a cylindrically multilayered nanostructure is based on classical electromagnetics with the quantum emitter modeled as a classical electric dipole radiating with a constant dipole moment (Lukosz, 1980; Sullivan $\mathcal{E}$ Hall, 1997). As a consequence of Fermi's golden rule, the radiative decay rate of the quantum emitter in the nanostructure is modified according to

$$
\Gamma_{r}=F \cdot \Gamma_{r}^{0}
$$

where $\Gamma_{r}^{0}$ and $\Gamma_{r}$ are the radiative decay rate in the bulk medium and the nanostructure, respectively. $F$ is the Purcell factor, which equals to the total radiation power of the dipole in the nanostruture normalized by the value in the bulk medium. Since the radiative decay and non-radiative decay are two competing processes, the quantum efficiency of the quantum emitter is modified in the nanostructure. Assuming that the non-radiative decay rate $\Gamma_{\mathrm{nr}}$ of the emitter does not change in the nanostructure, quantum efficiency $\eta_{m}$ in the nanostructure is changed to

$$
\eta_{m} \equiv \Gamma_{r} /\left(\Gamma_{r}+\Gamma_{n r}\right)=F \eta_{0} /\left[F \eta_{0}+\left(1-\eta_{0}\right)\right]
$$

where $\eta_{0}=\Gamma_{r}^{0} /\left(\Gamma_{r}^{0}+\Gamma_{n r}\right)$ is the quantum efficiency of the emitter in bulk. The spatial distribution of the emission, for example, the free space emission (emission goes to the far field) and the waveguide emission, can be obtained by evaluating the radiation property of the dipole source. Therefore, the radiation of a classical electric dipole in the cylindrically multilayered nanostructure basically reflects the emission properties of a quantum emitter in the structure. In principle, all the information can be obtained quite accurately by using a three-dimensional finite difference time domain (3D-FDTD) method (Bermel et al., 2004). However, the 3D-FDTD method is a brute force numerical method, which treats the problem as a black box and could not provide the physical insight. In addition, it is inefficient. Semi-analytical method based on cylindrical wave decomposition (Lovell \& Chew, $1987)$ is applicable. This method involves the evaluation of the so-called Sommerfeld-like integral and is inefficient for a direct evaluation. Here we propose a simple technique to efficiently calculate the Purcell factor $F$ and give an explicit formula to calculate the free space emission and the waveguide emission.

A cylindrically multilayered nanostructure is depicted in Fig. 1. An emitting layer is sandwiched between two stacks of shells, i.e., $P$ outer shells and $Q$ inner shells. The emitting medium and the outmost medium (e.g. air) are assumed to be non-absorbing at the emission wavelength while the other shells can be either transparent or absorptive. To study the spontaneous emission from the nanostructure, we assume the emitting layer consists of an ensemble of incoherent quantum emitters, each of which is modeled as an 
electric dipole radiating with a constant dipole moment. The orientation of the transition dipole moment of the emitter in a semiconductor nanostructure depends on the excitation. For the case of electroluminescence, the orientation is considered to be random while it is directed by the excitation for the case of PL. Here we investigate the emission of the emitters for three orthogonal orientations, namely, radially, azimuthally and $\mathrm{z}$ axially oriented. As displayed in Fig. 1, the emission from the emitter includes the waveguide emission $W$ (emission coupled to the waveguide modes) and the free space emission $U$ (emission coupled to the radiation modes). Here both of them are normalized by the total radiation power of the same dipole in the bulk medium. If all the materials are lossless, one has $F=U+W$.

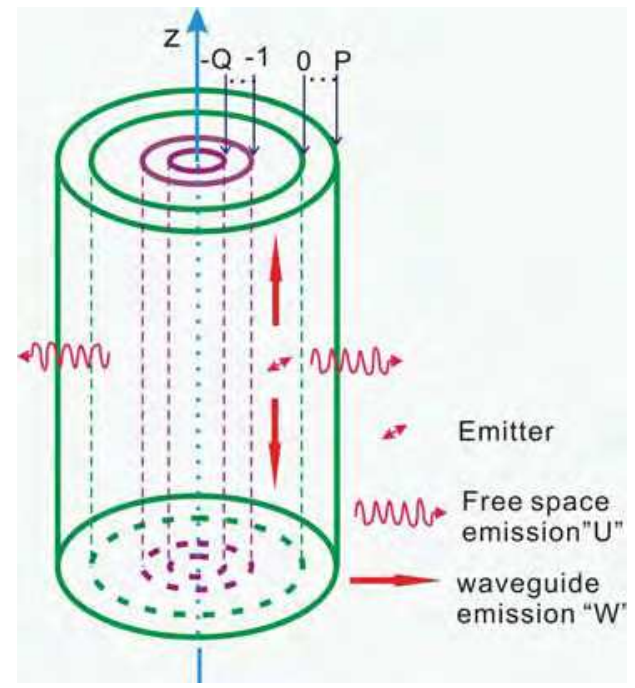

Fig. 1. Layout of a cylindrically multilayered structure. The spontaneous emission consists of free space emission and waveguide emission.

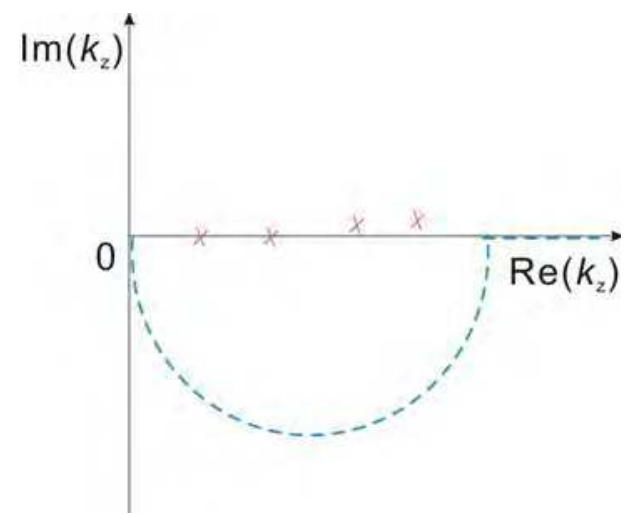

Fig. 2. Integration path in the complex plane of $k$. The crosses are the poles of the integral In the emitting layer of the nanostructure, the electric field induced by an electric dipole can be written as 


$$
E=E^{0}+E^{r}
$$

where $E^{0}$ and $E^{r}$ are the electric field of the dipole in the infinite medium and the reflected field due to the interfaces in the nanostructure, respectively. The normalized total emission power can be obtained as (Lukosz, 1980)

$$
F_{\alpha}=1-\vec{\alpha} \cdot \operatorname{Im}\left(i E^{r}\left(\vec{r}^{\prime}, \vec{r}^{\prime}\right)\right) /(\omega \mu k \Theta / 6 \pi)
$$

where $\mu, \omega, k, \Theta, \vec{\alpha}$, and $\vec{r}^{\prime}$ are the permeability, angular frequency, wavenumber in the emitting medium, dipole moment, orientation vector and location of the dipole, respectively. Here $\operatorname{Im}()$ stands for the imaginary part of () and the subscript of $F$ denotes the orientation. To calculate $F$, we expand the $\mathrm{z}$ component of the electric field $E^{0}$ and magnetic field $H^{0}$ in terms of cylindrical waves in polar coordinates $(\rho, \theta, z)($ Chew, 1995)

$$
\left[\begin{array}{c}
E_{z}^{0} \\
H_{z}^{0}
\end{array}\right]= \begin{cases}\sum_{v=-\infty}^{\infty} e^{i v \theta} \int_{-\infty}^{\infty} d k_{z} \vec{a}_{00 v} H_{v}^{(1)}\left(k_{\rho, e} \rho\right) e^{i k_{z} z} & \left(\rho>\rho^{\prime}\right) \\
\sum_{v=-\infty}^{\infty} e^{i v \theta} \int_{-\infty}^{\infty} d k_{z} \vec{b}_{00 v} J_{v}\left(k_{\rho, e} \rho\right) e^{i k_{z} z} & \left(\rho<\rho^{\prime}\right)\end{cases}
$$

where $k_{z}$ and $k_{\rho, e}$ are the $\mathrm{z}$ component and radial component of the wavenumber in the emitting medium. $\vec{a}_{00 v}$ and $\vec{b}_{00 v}$ are the coefficient vectors of outgoing wave and standing wave, respectively. They are given by

$$
\begin{aligned}
& \vec{a}_{00 v}=-\frac{\Theta}{8 \pi \varepsilon_{e} \omega}\left[\begin{array}{c}
\left(\vec{z} k^{2}+\frac{\partial}{\partial z^{\prime}} \nabla^{\prime}\right) \cdot \vec{\alpha} \\
i \omega \varepsilon_{e} \vec{\alpha} \cdot \vec{z} \times \nabla^{\prime}
\end{array}\right] e^{-i v \theta^{\prime}-i k_{z^{\prime}} z^{\prime}} J_{v}\left(k_{\rho, e} \rho^{\prime}\right) \\
& \vec{b}_{00 v}=-\frac{\Theta}{8 \pi \varepsilon_{e} \omega}\left[\begin{array}{c}
\left(\vec{z} k^{2}+\frac{\partial}{\partial z^{\prime}} \nabla^{\prime}\right) \cdot \vec{\alpha} \\
i \omega \varepsilon_{e} \vec{\alpha} \cdot \vec{z} \times \nabla^{\prime}
\end{array}\right] e^{-i v \theta^{\prime}-i k_{z} z^{\prime}} H_{v}^{(1)}\left(k_{\rho, e} \rho^{\prime}\right)
\end{aligned}
$$

Here $\varepsilon_{e}$ is the permittivity of the emitting layer. $J_{v}$ and $H_{v}^{(1)}$ denote the Bessel function and Hankel function of the first kind at order $v$. The operators with a prime act on the functions with primed coordinates. The coefficient vectors can be simplified for a specific dipole orientation. For the radially oriented dipole, the coefficient vectors read as

$$
\begin{gathered}
\bar{a}_{00 v}=\left[\begin{array}{c}
\frac{i k_{z} k_{\rho, e} \Theta}{8 \pi \omega \varepsilon_{e}} J_{v}^{\prime}\left(k_{\rho, e} \rho^{\prime}\right) \\
\frac{v \Theta}{8 \pi \rho^{\prime}} J_{v}\left(k_{\rho, e} \rho^{\prime}\right)
\end{array}\right] \\
\bar{b}_{00 v}=\left[\begin{array}{l}
\frac{i k_{z} k_{\rho, e}}{8 \pi \omega \varepsilon_{e}} H_{v}^{\prime}\left(k_{\rho, e} \rho^{\prime}\right) \\
\frac{v}{8 \pi \rho^{\prime}} H_{v}\left(k_{\rho, e} \rho^{\prime}\right)
\end{array}\right]
\end{gathered}
$$


For the azimuthally oriented dipole, the coefficient vectors read as

$$
\begin{gathered}
\bar{a}_{00 v}=\left[\begin{array}{c}
\frac{v k_{z}}{8 \pi \omega \varepsilon_{e} \rho^{\prime}} J_{v}\left(k_{\rho, e} \rho^{\prime}\right) \\
-\frac{i k_{\rho, e}}{8 \pi} J_{v}^{\prime}\left(k_{\rho, e} \rho^{\prime}\right)
\end{array}\right] \\
\bar{b}_{00 v}=\left[\begin{array}{c}
\frac{v k_{z}}{8 \pi \omega \varepsilon_{e} \rho^{\prime}} H_{v}\left(k_{\rho, e} \rho^{\prime}\right) \\
-\frac{i k_{\rho, e}}{8 \pi} H_{v}^{\prime}\left(k_{\rho, e} \rho^{\prime}\right)
\end{array}\right]
\end{gathered}
$$

For the z-axially oriented dipole, the coefficient vectors read as

$$
\begin{gathered}
\bar{a}_{00 v}=\left[\begin{array}{c}
-\frac{k^{2}-k_{z}^{2}}{8 \pi \omega \varepsilon_{e}} J_{v}\left(k_{\rho, e} \rho^{\prime}\right) \\
0
\end{array}\right] \\
\bar{b}_{00 v}=\left[\begin{array}{c}
-\frac{k^{2}-k_{z}^{2}}{8 \pi \omega \varepsilon_{e}} H_{v}\left(k_{\rho, e} \rho^{\prime}\right) \\
0
\end{array}\right]
\end{gathered}
$$

The fields in the $l$-th layer of the multilayered structure are combinations of the outgoing wave and standing wave and their $\mathrm{z}$ components can be given by

$$
\left[\begin{array}{c}
E_{z}^{l} \\
H_{z}^{l}
\end{array}\right]=\sum_{v=-\infty}^{\infty} e^{i v \theta} \int_{-\infty}^{\infty} d k_{z}\left(\vec{a}_{l v} H_{v}^{(1)}\left(k_{\rho, l} \rho\right)+\vec{b}_{l v} J_{v}\left(k_{\rho, l} \rho\right)\right) e^{i k_{z} z}
$$

The azimuthal components can be obtained from the $\mathrm{z}$ components and are expressed as

$$
\left[\begin{array}{c}
E_{\theta}^{l} \\
H_{\theta}^{l}
\end{array}\right]=\sum_{v=-\infty}^{\infty} e^{i v \theta} \int_{-\infty}^{\infty} d k_{z}\left(\vec{a}_{l v} \vec{H}_{v}^{(1)}\left(k_{\rho, l} \rho\right)+\vec{b}_{l v} \vec{J}_{v}\left(k_{\rho, l} \rho\right)\right) e^{i k_{z} z}
$$

where $\vec{H}_{v}^{(1)}$ and $\vec{J}_{v}$ are defined as

$$
\begin{gathered}
\vec{H}_{v}^{(1)}\left(k_{\rho} \rho\right)=\frac{i}{2 k_{\rho}}\left\{\left[\begin{array}{cc}
i k_{z} & -\omega \mu \\
\omega \varepsilon & i k_{z}
\end{array}\right] H_{v-1}^{(1)}\left(k_{\rho} \rho\right)+\left[\begin{array}{cc}
i k_{z} & -\omega \mu \\
-\omega \varepsilon & i k_{z}
\end{array}\right] H_{v+1}^{(1)}\left(k_{\rho} \rho\right)\right\} \\
\vec{J}_{v}\left(k_{\rho} \rho\right)=\frac{i}{2 k_{\rho}}\left\{\left[\begin{array}{cc}
i k_{z} & -\omega \mu \\
\omega \varepsilon & i k_{z}
\end{array}\right] J_{v-1}\left(k_{\rho} \rho\right)+\left[\begin{array}{cc}
i k_{z} & -\omega \mu \\
-\omega \varepsilon & i k_{z}
\end{array}\right] J_{v+1}\left(k_{\rho} \rho\right)\right\}
\end{gathered}
$$

The coefficient vectors of the neighbouring two layers are related according to the continuity of the tangentical field compoents.

$$
\vec{a}_{l v} H_{v}^{(1)}\left(k_{\rho, l} R_{l}\right)+\vec{b}_{l v} J_{v}\left(k_{\rho, l} R_{l}\right)=\vec{a}_{l+1 v} H_{v}^{(1)}\left(k_{\rho, l+1} R_{l}\right)+\vec{b}_{l+1 v} J_{v}\left(k_{\rho, l+1} R_{l}\right)
$$




$$
\vec{a}_{l v} \vec{H}_{v}^{(1)}\left(k_{\rho, l} R_{l}\right)+\vec{b}_{l v} \vec{J}_{v}\left(k_{\rho, l} R_{l}\right)=\vec{a}_{l+1 v} \vec{H}_{v}^{(1)}\left(k_{\rho, l+1} R_{l}\right)+\vec{b}_{l+1 v} \vec{J}_{v}\left(k_{\rho, l+1} R_{l}\right)
$$

where $R_{l}$ is the radial coordinate of the $l$-th interface of the multilayered structure. For the fields in the emitting layer $(l=0)$, the two stacks of shells can be considered as two "black" shells characterized by the total downward reflection matrix $N_{0 \mathrm{v}}$ and the total upward reflection matrix $M_{0 \mathrm{v}}$. The $\mathrm{z}$ components of the fields in the emitting layer due to the interfaces read as

$$
\left[\begin{array}{c}
E_{z}^{r} \\
H_{z}^{r}
\end{array}\right]=\sum_{v=-\infty}^{\infty} e^{i v \theta} \int_{-\infty}^{\infty} d k_{z}\left(\vec{a}_{0 v}^{r} H_{v}^{(1)}\left(k_{\rho, e} \rho\right)+\vec{b}_{0 v}^{r} J_{v}\left(k_{\rho, e} \rho\right)\right) e^{i k_{z} z}
$$

where the coefficient vectors are

$$
\begin{aligned}
\vec{a}_{0 v}^{r} & =\left[\vec{I}-M_{0 v} N_{0 v}\right]^{-1}\left[M_{0 v} \vec{b}_{00 v}+\vec{a}_{00 v}\right]-\vec{a}_{00 v} \\
\vec{b}_{0 v}^{r} & =\left[\ddot{I}-N_{0 v} M_{0 v}\right]^{-1}\left[N_{0 v} \vec{a}_{00 v}+\vec{b}_{00 v}\right]-\vec{b}_{00 v}
\end{aligned}
$$

The total upward reflection coefficient matrix $M_{0 \mathrm{v}}$ can be calculated recursively via (resulting from matching the boundary condition at each interface, Lovell \& Chew,1987)

$$
\begin{gathered}
\vec{M}_{l+1, v}=\vec{\Gamma}_{l, v}^{d}+\vec{T}_{l, v}^{u} \vec{M}_{l, v}\left[I-\vec{\Gamma}_{l, v}^{u} \vec{M}_{l, v}\right]^{-1} \vec{T}_{l, v}^{d} \quad(l=-Q, \ldots,-1) \\
\vec{M}_{-Q, v}=0
\end{gathered}
$$

where

$$
\begin{gathered}
\vec{T}_{l, v}^{d}=\frac{2 \omega}{\pi k_{\rho, k+1}^{2} R_{l}} \vec{A}_{l, v}\left[\begin{array}{cc}
0 & -\mu \\
\varepsilon_{l+1} & 0
\end{array}\right] \\
\vec{T}_{l, v}^{u}=\frac{2 \omega}{\pi k_{\rho, l}^{2} R_{l}} \vec{A}_{l, v}\left[\begin{array}{cc}
0 & -\mu \\
\varepsilon_{l} & 0
\end{array}\right] \\
\vec{\Gamma}_{l, v}^{d}=\vec{A}_{l, v}\left[J_{v}\left(k_{\rho, l} R_{l}\right) \vec{J}_{v}\left(k_{\rho, l+1} R_{l}\right)-J_{v}\left(k_{\rho, l+1} R_{l}\right) \vec{J}_{v}\left(k_{\rho, l} R_{l}\right)\right] \\
\vec{\Gamma}_{l, v}^{u}=\vec{A}_{l, v}\left[H_{v}^{(1)}\left(k_{\rho, l} R_{l}\right) \vec{H}_{v}^{(1)}\left(k_{\rho, l+1} R_{l}\right)-H_{v}^{(1)}\left(k_{\rho, l+1} R_{l}\right) \vec{H}_{v}^{(1)}\left(k_{\rho, l} R_{l}\right)\right] \\
\vec{A}_{l, v}=\left[H_{v}^{(1)}\left(k_{\rho, l+1} R_{l}\right) \vec{J}_{v}\left(k_{\rho, l} R_{l}\right)-J_{v}\left(k_{\rho, l} R_{l}\right) \vec{H}_{v}^{(1)}\left(k_{\rho, l+1} R_{l}\right)\right]^{-1}
\end{gathered}
$$

where $I$ is the identity matrix. Similarly, the total downward reflection matrix $N_{0 \mathrm{v}}$ can be obtained via

$$
\begin{gathered}
\vec{N}_{l, v}=\vec{\Gamma}_{l, v}^{u}+\vec{T}_{l, v}^{d} \vec{N}_{l+1, v}\left[I-\vec{\Gamma}_{l, v}^{d} \vec{N}_{l+1, v}\right]^{-1} \vec{T}_{l, v}^{u} \quad(l=P-1, P-2, . ., 0) \\
\vec{N}_{P, v}=\overrightarrow{0}
\end{gathered}
$$


After determining the coeffcient vectors in the emitting layer, the coefficient vectors in the outmost layer are obtained recursively by using Eq. (13). The $\mathrm{z}$ and azimuthal components of the fields in the outmost layer read

$$
\begin{gathered}
{\left[\begin{array}{c}
E_{z} \\
H_{z}
\end{array}\right]=\sum_{v=-\infty}^{\infty} e^{i v \theta} \int_{-\infty}^{\infty} d k_{z} \vec{a}_{P v} H_{v}^{(1)}\left(k_{\rho, P} \rho\right) e^{i k_{z} z}} \\
{\left[\begin{array}{c}
E_{\theta} \\
H_{\theta}
\end{array}\right]=\sum_{v=-\infty}^{\infty} e^{i v \theta} \int_{-\infty}^{\infty} d k_{z}\left(\vec{a}_{P v} \vec{H}_{v}^{(1)}\left(k_{\rho, P} \rho\right)\right) e^{i k_{z} z}}
\end{gathered}
$$

\subsection{Efficient evaluation of the Purcell factor and free space emission}

As seen from Eq. (4) and Eq. (14), the calculation of Purcell factor F involves the evaluation of an integral with pole singularities, which physically correspond to the guided modes in the cylindrically layered structure. This is similar to the case of a planar multilayered structure (Gay-Balmaz \& Mosig, 1997; Chen et al, 2007b) and a direct evaluation of the integral in Eq. (14) tends to fail. These poles are located in the real axis of $k_{\mathrm{z}}$ or in the first quadrant of the complex plane of $k_{z}$. Here, to get round the problem, we change the 1D integral of Eq. (14) in the real space to a line integral in the complex plane of $k_{\mathrm{z}}$ and use the Cauchy integral theorem to perform the integration. Since the poles of the integrand in Eq. (14) are located in the first quadrant of the complex plane of $k_{z}$, we select an integration path in the fourth quadrant such that the domain enclosed by the new path and the real axis of $k_{z}$ does not contain any pole. The new path shown by the dashed line in Fig. 2 consists of a semicircle in the fourth quadrant of the complex plane and a straight line along the real axis. The integral in Eq. (14) can be rewritten as

$$
\left[\begin{array}{c}
E_{z}^{r} \\
H_{z}^{r}
\end{array}\right]=2 \sum_{v=-\infty}^{\infty} e^{i v \theta}\left(\int_{C_{R}}+\int_{2 k_{r}}^{\infty}\right)\left(\vec{a}_{0 v} H_{v}^{(1)}\left(k_{\rho, e} \rho\right)+\vec{b}_{0 v} J_{v}\left(k_{\rho, e} \rho\right)\right) e^{i k_{z} z} d k_{z}
$$

where $k_{\mathrm{r}}$ is the radius of the semicircle $C_{R}$. $k_{\mathrm{r}}$ should be large enough so that the semicircle can bypass all the poles of the integrand. In this way, the integration can be efficiently evaluated by using conventional numerical integration routines such as the Gaussian quadrature technique (Chen et al, 2007b).

The free space emission power $U$ is the power leaving the cylindrically layered structure from the lateral surface to far field. Thus, $U$ can be expressed as

$$
U \equiv \int_{0}^{2 \pi} \rho d \theta \int_{-\infty}^{\infty} d z \frac{1}{2} \operatorname{Re}\left(E_{\theta} \times H_{z}^{*}-E_{z} \times H_{\theta}^{*}\right) / P_{0}
$$

where $P_{0}=\sqrt{\mu / \varepsilon_{e}} k_{e}^{2} /\left(48 \pi^{3}\right)$ is the normalization constant and $\operatorname{Re}()$ stands for the real part of (). By using Eqs. (21) and (22), Eq.(24) can be written as

$$
U=\frac{\omega \rho}{P_{0}} \sum_{v=-\infty}^{\infty} \int_{0}^{k_{o u t}} d k_{z} \operatorname{Re}\left[i \varepsilon_{P} H_{v}^{(1)}\left(k_{\rho, P} \rho\right) H_{v}^{\prime(1) *}\left(k_{\rho, P} \rho\right)\left|a_{P_{v}}(1)\right|^{2}-i \mu H_{v}^{(1) *}\left(k_{\rho, P} \rho\right) H_{v}^{\prime(1)}\left(k_{\rho, P} \rho\right)\left|a_{P v}(2)\right|^{2}\right] / k_{\rho, P}
$$


Here $\varepsilon_{\mathrm{P}}$ and $k_{\mathrm{P}}$ are the permittivity and wavenumber of the outmost medium, respectively. If the outermost medium is lossless, $U$ does not depend on $\rho$. If the whole structure is lossless, the waveguide emission $W$ can be readily obtained by $W=F-U$.

\subsection{Examples: Spontaneous emission from a single ZnO NW and a ZnO NT}

In this section, we employ the optical model developed above to study the spontaneous emission from a single $\mathrm{ZnO} \mathrm{NW}$ and a ZnO NT. Light emission properties, including the radiative decay rate, the quantum efficiency and the fraction of the waveguide emission to the total emission, are investigated as a function of the size of the NW and NT at an emission wavelength of $387 \mathrm{~nm}$. The cross-section of the ZnO NW is treated approximately as circular here and an initial quantum efficiency $\eta_{0}=0.85$ is used (Zhang \& Russo, 2005). A refractive index of 2.5 is used for the $\mathrm{ZnO}$ material here.
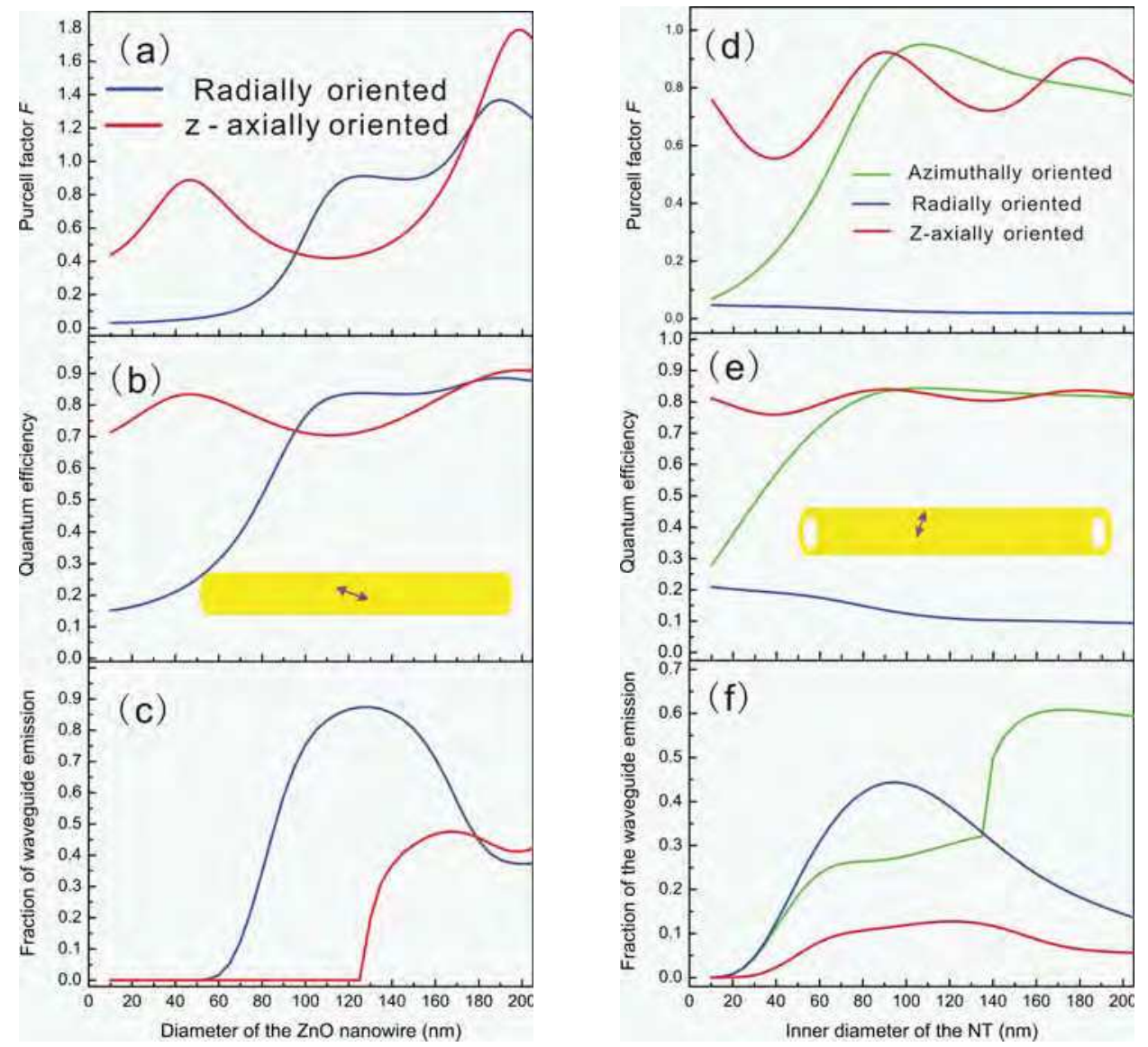

Fig. 3. Spontaneous emission properties of a single $\mathrm{ZnO} N W(\mathrm{a}-\mathrm{c}$ ) and a single ZnO NT (d-f)

Figures 3(a)-(c) show the spontaneous emission properties of an emitter as a function of the $\mathrm{ZnO} \mathrm{NW}$ diameter. The emitter is on the $\mathrm{z}$ axis and two kinds of orientations, namely the 
radial and z-axial orientations are considered in the simulation. Several remarkable features can be observed in these figures. Firstly, the Purcell factor of the radially oriented emitter is almost one order of magnitude smaller than the value of the z-axially oriented emitter for ZnO NWs with a small diameter. As the diameter of the NW decreases further, $F_{\rho}$ has a limiting value of 0.03 , which solely depends on the refractive index of the $\mathrm{ZnO}$ material. Since the radiative decay rate is proportional to $F$ (c.f. Eq. (1)) and radiative transition and non-radiative transition are two competing processes, the quantum efficiency of the radially oriented emitter is significantly lower than the value of the $\mathrm{z}$ axially oriented emitter, as shown in Fig. 3(b). As the NW diameter increases, $F_{\rho}$ goes up quickly and reaches a value close to $F_{z}$ and then starts to oscillate, similar to $F_{z}$. Secondly, there are two critical diameters of the NW, i.e. $60 \mathrm{~nm}$ and $125 \mathrm{~nm}$ for the radially oriented emitter and $z$-axially oriented emitter, respectively. For NWs thinner than $60 \mathrm{~nm}$, as seen in Fig. 3(c), there is no waveguide emission for both kinds of emitters. But for NWs thicker than $60 \mathrm{~nm}$ but thinner than $125 \mathrm{~nm}$, the z-axially oriented emitter has almost zero waveguide emission. Here a larger critical diameter for the $z$ axially oriented emitter is due to the fact that the emission from the $z$-axially oriented emitter located on the $\mathrm{z}$ axis can only couple to $\mathrm{TM}_{01}$ waveguide mode because the coefficient vectors in Eq.(9a) are nonzero only for $n=0$. TM01 mode is not the fundamental mode and is cut-off until the diameter is larger than $125 \mathrm{~nm}$. However, the radially oriented emitter has a good coupling to the waveguide modes starting from the fundamental mode, i.e., $\mathrm{HE}_{11}$ mode. For the $\mathrm{NW}$ thinner than $60 \mathrm{~nm}$, the emission is purely free space emission and the z-axially oriented emitter is much more efficient than the radially oriented emitter (see Fig. 3(b)). As the diameter increases from $60 \mathrm{~nm}$ to $125 \mathrm{~nm}$, the emission efficiency of the radially oriented emitter steadily increases and the waveguide emission gradually becomes dominant.

Figures 3(d)-(f) show the Purcell factor, quantum efficiency and fraction of waveguide emission as a function of the inner diameter of a ZnO NT with a shell thickness of $20 \mathrm{~nm}$. The emitter sits in the middle of the $\mathrm{ZnO}$ shell and can have three orthogonal orientations, namely, the radial, azimuthal and $\mathrm{z}$ axial orientations. The emission properties are distinct for different orientations. For the ZnO NT studied here, $F_{\rho}$ is always the smallest and decreases as the inner diameter of the NT increases. Thus the radially oriented emitter is the most inefficient one. $F_{z}$ oscillates as a function of the inner diameter and is much larger than $F_{\theta}$ and $F_{\rho}$ for the NTs with small inner diameters. As the inner diameter increases, $F_{\theta}$ goes up quickly to a maximum value of 0.95 and then decreases slowly. Figure 3(e) shows the quantum efficiencies of the three kinds of emitters. As the inner diameter increases from 10 $\mathrm{nm}$ to $80 \mathrm{~nm}$, the $\mathrm{z}$ axially oriented emitter remains the most efficient one while the efficiency of the azimuthally oriented emitter increases from $28 \%$ to $81 \%$. As the diameter further increases, the quantum efficiencies of the above two emitters become similar and remain above $80 \%$. For the waveguide emission, different from the case of NW, there is no critical inner diameter below which no waveguide emission exists.

In summary, we have presented an efficient and accurate optical model of spontaneous emission from a single cylindrically multilayered nanostructure. The Purcell factor is represented as an integral and can be efficiently evaluated by selecting a new integration path in the complex plane. An explicit formula has been derived to calculate the free space emission. As examples of practical interest, the emission properties of a single $\mathrm{ZnO} \mathrm{NW}$ and a ZnO NT are comprehensively studied and discussed. We found that the emission properties depend strongly on the orientation of the emitter and consequently the PL should show strong dependence on the polarization state of the excitation light. 


\section{Photoluminescence of cylindrically multilayered nanostructure}

In section 2, we studied the spontaneous emission of a single emitter with a given orientation in a cylindrically multilayered structure without considering the effect of excitation. For PL of the semiconductor nanostructure, the excitation light determines the spatial distribution of the density of the excitons and the orientation of the transition dipole moment, and therefore governs the emission of the nanostructure. The exciton density in the semiconductor nanostructure is proportional to the local intensity of excitation light $I_{e x}$ and the orientation of the dipole moment is directed by the local electric field. Therefore, the total emission intensity $I_{\mathrm{t}}$ of a cylindrically multilayered nanostructure can be expressed as

$$
I_{t}=\int_{r_{n}}^{r_{n+1}} \rho d \rho \int_{0}^{2 \pi} d \theta\left[I_{e x, \rho} \eta_{\rho}+I_{e x, \theta} \eta_{\theta}+I_{e x, z} \eta_{z}\right] /\left(\pi r_{n+1}^{2}-\pi r_{n}^{2}\right)
$$

where the subscript $\mathrm{n}$ denotes the emitting layer which is between two interfaces labeled by $r_{\mathrm{n}}$ and $r_{\mathrm{n}+1}$ respectively. $\eta_{x}(x=\rho, \theta, z)$ is the quantum efficiency of the emitter oriented along $x$ direction while $I_{e x, x}$ is the intensity of the excitation along $x$ direction at the position of the emitter. The free space emission is given by

$$
I_{u}=\int_{r_{n}}^{r_{n+1}} \rho d \rho \int_{0}^{2 \pi} d \theta\left[I_{e x, \rho} \eta_{\rho} \frac{U_{\rho}}{F_{\rho}}+I_{e x, \theta} \eta_{\theta} \frac{U_{\theta}}{F_{\theta}}+I_{e x, z} \eta_{z} \frac{U_{z}}{F_{z}}\right] /\left(\pi r_{n+1}^{2}-\pi r_{n}^{2}\right)
$$

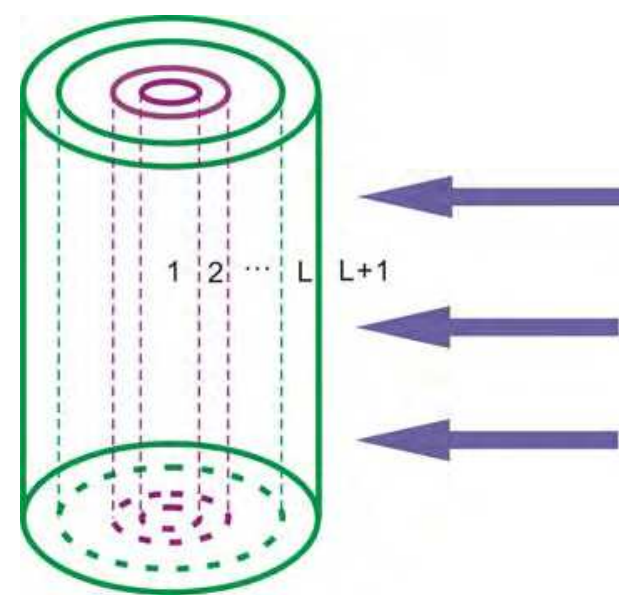

Fig. 4. Schematic diagram of a cylindrically multilayered structure illuminated by a plane wave.

By following the model in Section 2, one could easily evaluate $\eta_{x}, U_{x}$ and $F_{x}$ at arbitrary position. For a lossless structure, the waveguide emission is $I_{\mathrm{t}}-I_{\mathrm{u}}$. In this section, we first introduce how to calculate the local excitation field by a plane wave excitation and then simulate the PL from a single $\mathrm{ZnO}$ NW, NC and NT. 


\subsection{Modeling of the excitation}

In this subsection, we consider a single cylindrically multilayered nanostructure illuminated by a polarized plane wave and formulate the problem to calculate the electric field at an arbitrary position in the structure. In polar coordinates, an arbitrary polarized plane wave can be decomposed into two polarizations, namely the vertical polarization with electric field parallel to the $\mathrm{z}$ axis and the horizontal polarization with electric field perpendicular to the $\mathrm{z}$ axis, each of which can be expanded in terms of cylindrical waves with coefficients determined by the wave vector and amplitude of the plane wave. By summing up the response of the nanostructure from each cylindrical wave, one obtains the response of the nanostructure by the plane wave. A plane wave $E_{i}$ is decomposed as the following (Tsang et al., 2001)

$$
E_{i}(\vec{r})=\left(\widehat{v}_{i} E_{v i}+\widehat{h}_{i} E_{h i}\right) e^{i \bar{k} \cdot \vec{r}}=\sum_{n=-\infty}^{\infty} \frac{-i^{n} e^{-i n \theta_{k}}}{k_{\rho}}\left[E_{v i} \bar{N}_{n}^{J}\left(k_{\rho}, k_{z}, \vec{r}\right)-i E_{h i} \bar{M}_{n}^{J}\left(k_{\rho}, k_{z}, \vec{r}\right)\right]
$$

where $\theta_{k}$ is the azimuthal angle of the incident wave vector $\vec{k}$, subscript $v$ and $h$ denote the vertical and horizontal components, respectively. $k_{\rho}$ and $k_{z}$ are respectively the radial and $\mathrm{z}$ axial components of the wave vector of the incident plane wave. The cylindrical vector wave functions are defined as

$$
\begin{gathered}
\vec{M}_{n}^{X}\left(k_{\rho}, k_{z}, \vec{r}\right)=\left(\hat{\rho} \frac{i n}{\rho} X_{n}\left(k_{\rho} \rho\right)-\hat{\theta} k_{\rho} X_{n}^{\prime}\left(k_{\rho} \rho\right)\right) e^{i k_{z} z+i n \theta} \\
\bar{N}_{n}^{X}\left(k_{\rho}, k_{z}, \bar{r}\right)=\left(\hat{\rho} \frac{i k_{\rho} k_{z}}{k} X_{n}^{\prime}\left(k_{\rho} \rho\right)-\hat{\theta} \frac{n k_{z}}{k \rho} X_{n}\left(k_{\rho} \rho\right)+\widehat{z} \frac{k_{\rho}^{2}}{k} X_{n}\left(k_{\rho} \rho\right)\right) e^{i k_{z} z+i n \theta}
\end{gathered}
$$

where $X_{n}$ denotes the bessel function (if $X=J$ ) or the Hankel function of first kind (if $X=H$ ) at order $n$. These cylindrical vector wave functions satisfy the following relations

$$
\begin{aligned}
& \nabla \times \vec{M}_{n}^{X}\left(k_{\rho}, k_{z}, \vec{r}\right)=k \vec{N}_{n}^{X}\left(k_{\rho}, k_{z}, \vec{r}\right) \\
& \nabla \times \vec{N}_{n}^{X}\left(k_{\rho}, k_{z}, \vec{r}\right)=k \vec{M}_{n}^{X}\left(k_{\rho}, k_{z}, \vec{r}\right)
\end{aligned}
$$

In the $l$-th layer of the nanostructure shown in Fig. 4, the electric field and magnetic field are given by

$$
\begin{gathered}
\vec{E}_{l}(\vec{r})=\sum_{n=-\infty}^{\infty}\left[\begin{array}{l}
A_{l, n}^{M} \vec{M}_{n}^{J}\left(k_{l, \rho}, k_{z}, \vec{r}\right)+A_{l, n}^{N} \vec{N}_{n}^{J}\left(k_{l, \rho}, k_{z}, \vec{r}\right)+ \\
B_{l, n}^{M} \vec{M}_{n}^{H}\left(k_{l, \rho}, k_{i z}, \vec{r}\right)+B_{l, n}^{N} \vec{N}_{n}^{H}\left(k_{l, \rho}, k_{z}, \vec{r}\right)
\end{array}\right] \\
H_{l}=\frac{i}{\omega \mu} \nabla \times E_{l}=\frac{i k_{l}}{\omega \mu} \sum_{n=-\infty}^{\infty}\left[\begin{array}{l}
A_{l, n}^{M} \vec{N}_{n}^{J}\left(k_{l, \rho}, k_{z}, \vec{r}\right)+B_{l, n}^{M} \vec{N}_{n}^{H}\left(k_{l, \rho}, k_{z}, \vec{r}\right) \\
+A_{l, n}^{N} \vec{M}_{n}^{J}\left(k_{l, \rho}, k_{z}, \vec{r}\right)+B_{l, n}^{N} \vec{M}_{n}^{H}\left(k_{l, \rho}, k_{z}, \vec{r}\right)
\end{array}\right]
\end{gathered}
$$

where $A_{l, n}^{M}, A_{l, n}^{N}, B_{l, n}^{M}$ and $B_{l, n}^{N}$ are the coefficients of the cylindrical vector wave functions. The coefficients between the $l$-th layer and $l+1$-th layer are related through the boundary condition, i.e. the continuity of the tangential field components, which are given by 


$$
\begin{gathered}
A_{l+1}^{M} k_{l+1, \rho} J_{n}{ }^{\prime}\left(k_{l+1, \rho} r_{l}\right)+B_{l+1}^{M} k_{l+1, \rho} H_{n}^{(1)}{ }^{\prime}\left(k_{l+1, \rho} r_{l}\right)+A_{l+1}^{N} \frac{n k_{z}}{k_{l+1} r_{l}} J_{n}\left(k_{l+1, \rho} r_{l}\right)+B_{l+1}^{N} \frac{n k_{z}}{k_{l+1} r_{l}} H_{n}^{(1)}\left(k_{l+1, \rho} r_{l}\right) \\
=A_{l}^{M} k_{l, \rho} J_{n}{ }^{\prime}\left(k_{l, \rho} r_{l}\right)+B_{l}^{M} k_{l, \rho} H_{n}^{(1)}{ }^{\prime}\left(k_{l, \rho} r_{l}\right)+A_{l}^{N} \frac{n k_{z}}{k_{l} r_{l}} J_{n}\left(k_{l, \rho} r_{l}\right)+B_{l}^{N} \frac{n k_{z}}{k_{l} r_{l}} H_{n}^{(1)}\left(k_{l, \rho} r_{l}\right) \\
A_{l+1}^{N} \frac{k_{l+1, \rho}^{2}}{k_{l+1}} J_{n}\left(k_{l+1, \rho} r_{l}\right)+B_{l+1}^{N} \frac{k_{l+1, \rho}^{2}}{k_{l+1}} H_{n}^{(1)}\left(k_{l+1, \rho} r_{l}\right)=A_{l}^{N} \frac{k_{l, \rho}^{2}}{k_{l}} J_{n}\left(k_{l, \rho} r_{l}\right)+B_{l}^{N} \frac{k_{l, \rho}^{2}}{k_{l}} H_{n}^{(1)}\left(k_{l, \rho} r_{l}\right) \\
A_{l+1}^{M} k_{l+1, \rho}^{2} J_{n}\left(k_{l+1, \rho} r_{l}\right)+B_{l+1}^{M} k_{l+1, \rho}^{2} H_{n}^{(1)}\left(k_{l+1, \rho} r_{l}\right)=A_{l}^{M} k_{l, \rho}^{2} J_{n}\left(k_{l, \rho} r_{l}\right)+B_{l}^{M} k_{l, \rho}^{2} H_{n}^{(1)}\left(k_{l, \rho} r_{l}\right) \\
=k_{l}\left[-A_{l}^{N} k_{l+1, \rho} J_{n}{ }^{\prime}\left(k_{l+1, \rho}{ }^{\prime}{ }_{n}\right)-B_{l+1}^{N} k_{l, \rho} r_{l}\right)-B_{l+\rho}^{N} k_{l, \rho} H_{n}^{(1)} H_{n}^{(1)}\left(k_{l+1, \rho}\left(k_{l, \rho} r_{l}\right)-A_{l}^{M} \frac{n k_{z}}{k_{l} r_{l}} J_{n}\left(k_{l+1} \frac{n k_{z}}{k_{l+1} r_{l}} J_{n}\left(k_{l+1, \rho} r_{l}\right)-B_{l}^{M} \frac{n k_{z}}{k_{l} r_{l}} H_{n}^{(1)}\left(k_{l, \rho} r_{l}\right)\right]\right.
\end{gathered}
$$

where the subscript $n$ of the coefficients is droped for simplicity. The coefficients in the outmost and innermost layers satisfy the following the conditions.

$$
\begin{gathered}
A_{L+1, n}^{M}=\frac{i^{n} e^{-i n \phi_{k}}}{k_{L+1, \rho}} i E_{h i} \quad A_{L+1, n}^{N}=-\frac{i^{n} e^{-i n \phi_{k}}}{k_{L+1, \rho}} E_{v i} \\
B_{1, n}^{M}=0 \quad B_{1, n}^{N}=0
\end{gathered}
$$

By using Eq.(35) - (40), all the coefficients can be readily determined and consequently the electric field at any position in the nanostructure can be computed according to Eq. (33).

\subsection{Results and discussion}

In this subsection, we employ the approach introduced in Section 3.1 and the spontaneous emission model developed in Section 2 to respectively investigate the PL of a single $\mathrm{ZnO}$ $\mathrm{NW}, \mathrm{ZnO} \mathrm{NC}$ and ZnO NT illuminated by a plane wave at a wavelength of $325 \mathrm{~nm}$. The wave vector is in the horizontal plane (having no $\mathrm{z}$ component) and two kinds of polarizations, namely, the vertical and horizontal polarizations, are considered in the simulation. The ZnO nanostructures emit light spontaneously at $387 \mathrm{~nm}$.

Figures 5 (a) and (b) show the PL intensity of the ZnO NW as a function of the diameter for two kinds of polarizations. In the figures, one critical diameter, i.e. $70 \mathrm{~nm}$, is observed. For the NWs thinner than $70 \mathrm{~nm}$, the waveguide emission is negligible for both kinds of polarizations. The critical diameter obtained previously from the spontaneous emission model is larger than $60 \mathrm{~nm}$ because the PL is the product of the quantum efficiency and excitation field (c.f. Eq. (26)). The latter is weak for the horizontally polarized illumination as seen in Fig. 5(b). The second critical diameter does not show up in Fig. 5(a) because the emission by the emitters sitting away from $\mathrm{z}$ axis has nonzero coupling to the $H E_{11}$ mode.

For thin NWs, PL by the excitation with a vertical polarization is by tens of times larger than the emission from the excitation with a horizontal excitation. As the diameter of the NW increases, PL from two kinds of excitation becomes comparable and considerable amount of 
waveguide emissions appear. These properties should be interesting for the applications of nanowire LEDs and nanolasers.
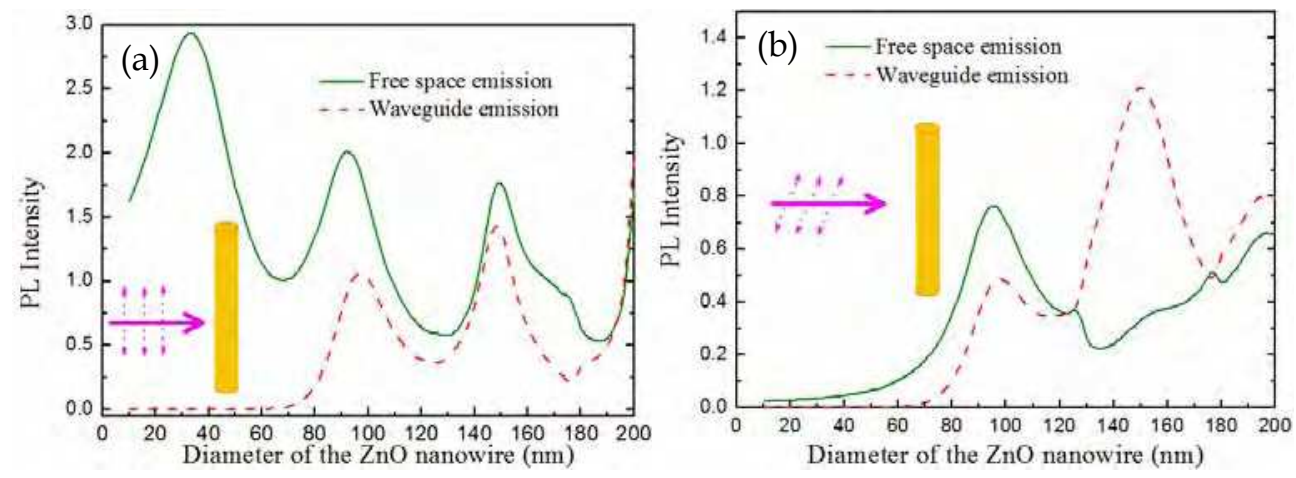

Fig. 5. PL as a function of the ZnO NW diameter (a) excitation field polarized vertically excitation field polarized horizontally
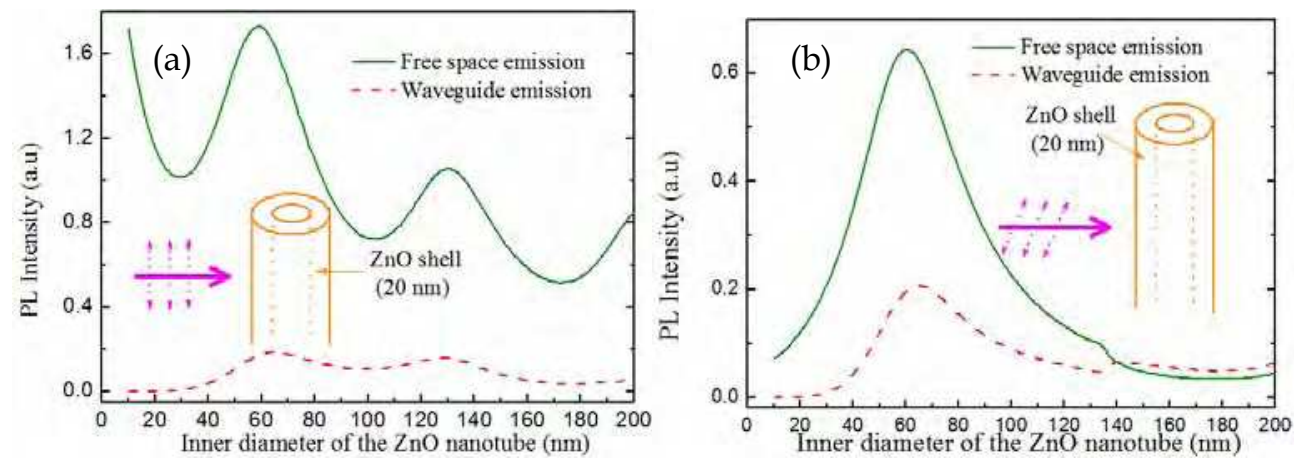

Fig. 6. PL as a function of the inner diameter of the ZnO NT diameter (a) excitation field polarized vertically (b) excitation field polarized horizontally

Figures 6(a) and (b) depict the PL of a single ZnO NT for two kinds of polarizations. For thin NTs, the free space emission is always larger than the waveguide emission. The PL by the excitation with a horizontal polarization is much weaker than the emission by the vertically polarized excitation. At an inner diameter of $65 \mathrm{~nm}$, PLs, including free space emission and waveguide emission, show a maximum for both kinds of excitation.

Figures 7(a) and (b) display the PL of a single $\mathrm{ZnO} \mathrm{NC}$ (i.e. a $20 \mathrm{~nm} \mathrm{ZnO}$ core with a silica shell) as a function of the thickness of the silica shell. As observed from the Figures, the PL in (a) is larger than the value in (b) by more than an order of magnitude. For vertically polarized excitation in Fig. 7(a), the free space emission always dominates. Since the excitons are confined in the $20 \mathrm{~nm} \mathrm{ZnO}$ core, there are two different critical values of the shell thickness, below which no waveguide emission exists, for excitations with different polarizations. For the excitation with vertical polarization, the critical shell thickness is 120 $\mathrm{nm}$ while it is $40 \mathrm{~nm}$ for the excitation with horizontal polarization. 

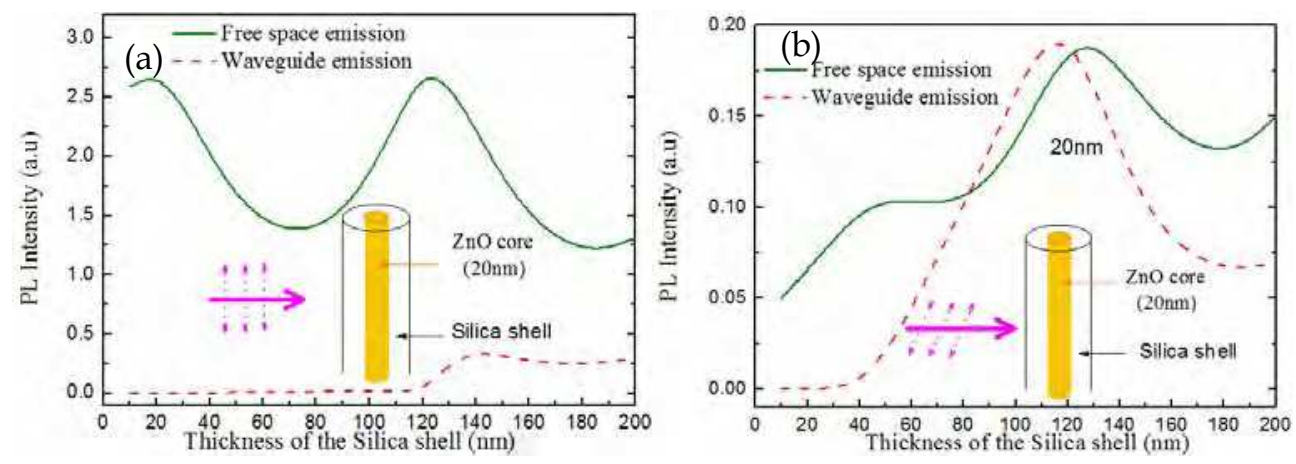

Fig. 7. PL as a function of the thickness of the silica shell of the ZnO NC (a) excitation field polarized vertically (b) excitation field polarized horizontally

\section{Conclusion}

In conclusion, we have presented an efficient and accurate optical model for studying the PL of a single cylindrically multilayered nanostructure. The model consists of two parts, namely, the modeling of the spontaneous emission of a single emitter in the nanostructure at the emission wavelength and the evaluation of the excitation effect at the illumination wavelength. As examples of practical interest, we have studied the PL of a single $\mathrm{ZnO}$ nanowire, $\mathrm{ZnO}$ nanotube and $\mathrm{ZnO} /$ Silica nanocable under plane wave illumination with two orthogonal polarization states. We found that the PL is highly excitation polarization dependent and the polarization dependence is stronger for thinner nanowires, nanotubes and nanocables. The free space emission and waveguide emission have been calculated as a function of the size of the nanostructure and the polarization state of the excitation. The optical model and the results should be useful and interesting for designing onedimensional semiconductor active nanostructure for various applications.

\section{Acknowledgement}

This work is supported partially by the National Natural Science Foundation of China under grant number 60688401. One of the authors (X.-W. Chen) would like to thank Wallace C. H. Choy for helpful discussions and encouragement during the author's stay at the University of Hong Kong.

\section{References}

Bao J.M., Zimmler M.A., Capasso F., Wang X.W., Ren Z. F. (2006). Broadband ZnO singlenanowire light-emitting diode, Nano Lett. Vol.6, No. 8, 1719-1722, ISSN 1530-6984.

Barrelet C.J., Greytak A.B., Lieber C.M. (2004). Nanowire photonic circuit elements. Nano Lett. Vol. 4, No. 10, 1981-1985, ISSN 1530-6984.

Bermel P., Joannopoulos J.D., Fink Y. (2004) Properties of radiating pointlike sources in cylindrically omnidirectionally reflecting waveguides. Phys. Rev. B Vol. 69, No. 3, 035316, ISSN 0163-1829. 
Chen X.W., Choy W.C.H., He S.L. (2007a) Efficient optical modeling of spontaneous emission in a cylindrically layered nanostructure. Opt. Express Vol. 15, No. 16, 10356-10361, ISSN 1094-4087.

Chen X.W., Choy W.C.H., He S.L., Chui P.C. (2007b) Efficient and Rigorous Modeling of Light Emission in Planar Multilayer Organic Light-Emitting Diodes. IEEE/OSA, Journal of Display Technolog, Vol. 3, No. 2, 110-117, ISSN 1551-319X.

Chew W.C. (1995). Waves and Fields in Inhomogeneous Media. IEEE Press, ISBN 0780311167, New York.

Duan X.F., Huang Y., Agarwall R., Lieber C.M. (2003). Single-nanowire electrically driven lasers. Nature Vol. 421, (16 January 2003), 241-245, ISSN 0028-0836.

Gay-Balmaz P., Mosig J.R.(1997). Three-Dimensional planar radiating structures in stratified media. Int. J. Microwave Millimeter Wave Computer-Aided Eng. Vol. 37, 330-343, ISSN. 1050-1827.

Huang M. H., Mao S., Feick H., Yan H.Q., Wu Y.Y., Kind H., Weber E., Russo R., Yang P.D. (2001). Room-temperature ultraviolet nanowire nanolasers. Science Vol. 292. No. 5523, 1897 - 1899 ISSN 0036-8075.

Johnson J.C., Choi H.J., Knutsen K.P., Schaller R.D., Yang P.D., Saykally R.J. (2002). Single gallium nitride nanowire lasers. Nature Materials Vol. 1, (15 September 2002), 106110, ISSN 1476-1122.

Johnson J.C., Yan H.Q., Yang P.D., Saykally R.J. (2003). Optical cavity effects in ZnO nanowire lasers and waveguides. J. Phys. Chem. B, Vol. 107, No. 34, 8816-8828, ISSN 1089-5647.

Könenkamp R., Word R. C., and Schlegel C. (2004). Vertical nanowire light-emitting diode. Appl. Phys. Lett. Vol. 85, No.24 , 6004-6006, ISSN 0003-6951.

Lovell J. R., Chew W.C. (1987) Response of a point source in a multicylindrically layered medium. IEEE Trans. Geosci. Remote Sensing,Vol. GE-25,No.6, 850-858, ISSN 01962892.

Lukosz W. (1980). Theory of optical-environment-dependent spontaneous emission rates for emitters in thin layers. Phys. Rev. B Vol. 22, No. 6, 3030-3038, ISSN 1098-0121.

Maslov, A. V., Ning, C. Z. (2003). Reflection of guided modes in a semiconductor nanowire laser. Appl. Phys. Lett., Vol. 83, No. 6, 1237-1239, ISSN 0003-6951.

Purcell E.M. (1946), Spontaneous emission probabilities at radio frequencies. Phys. Rev. Vol. $69,681$.

Qian F., Gradecak S., Li Y., Wen C.Y., Lieber C.M. (2005) Core/multishell nanowire heterostructures as multicolor, high-efficiency light-emitting diodes. Nano Lett. Vol. 5, No. 11, 2287-2291, ISSN 1530-6984.

Sullivan K.G. \& Hall D.G.(1997). Enhancement and inhibition of electromagnetic radiation in plane-layered media. I. Plane-wave spectrum approach to modeling classical effects. J. Opt. Soc. Am. B, Vol. 14, No. 5, 1149-1159, ISSN 0740-3224.

Tong L.M., Lou J.Y., Gattass R.R., He S.L., Chen X.W., Liu L. Mazur E. (2005). Assembly of silica nanowires on silica aerogels for microphotonic devices, Nano Lett. Vol. 5, No. 2, 259-262, ISSN 1530-6984.

Tsang L., Kong J.A., Ding K.H. (2001), Scattering of Electromagnetic Waves: Theories and Applications, Wiley-Interscience, ISBN 0471387991, Boston. 
Wang Y., Tang Z.Y., Kotov N.A. (2005). Bioapplication of nanosemiconductors, Materials Today Vol. 8, No. 5 (Supplement 1, May 2005), 20-31, ISSN 1369-7021.

Wang J.F., Gudiksen M.S., Duan X.F., Cui Y., Lieber C.M. (2001). Highly Polarized Photoluminescence and Photodetection from Single Indium Phosphide Nanowires. Science Vol. 293, No. 5534 1455-1457, ISSN 0036-8075.

Yu D. P., Hang Q. L., Ding Y., Zhang H. Z., Bai Z. G., Wang J. J., Zou Y. H., Qian W., Xiong G. C., and Feng S. Q. (1998). Amorphous silica nanowires: Intensive blue light emitters. Appl. Phys. Lett. Vol. 73, No. 21, 3076-3078, ISSN 0003-6951.

Zhang Y. Russo R.E. (2005). Quantum efficiency of ZnO nanowire nanolasers. Appl. Phys. Lett. Vol. 87, No.4, 043106-043108, ISSN 0003-6951. 


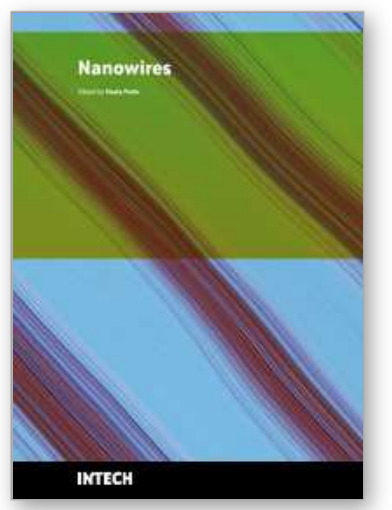

\author{
Nanowires \\ Edited by Paola Prete
}

ISBN 978-953-7619-79-4

Hard cover, 414 pages

Publisher InTech

Published online 01, February, 2010

Published in print edition February, 2010

This volume is intended to orient the reader in the fast developing field of semiconductor nanowires, by providing a series of self-contained monographs focusing on various nanowire-related topics. Each monograph serves as a short review of previous results in the literature and description of methods used in the field, as well as a summary of the authors recent achievements on the subject. Each report provides a brief sketch of the historical background behind, the physical and/or chemical principles underlying a specific nanowire fabrication/characterization technique, or the experimental/theoretical methods used to study a given nanowire property or device. Despite the diverse topics covered, the volume does appear as a unit. The writing is generally clear and precise, and the numerous illustrations provide an easier understanding of the phenomena described. The volume contains 20 Chapters covering altogether many (although not all) semiconductors of technological interest, starting with the IV-IV group compounds ( $\mathrm{SiC}$ and SiGe), carrying on with the binary and ternary compounds of the III-V (GaAs, AIGaAs, GaSb, InAs, GaP, InP, and GaN) and II-VI ( $\mathrm{HgTe}, \mathrm{HgCdTe}$ ) families, the metal oxides ( $\mathrm{CuO}, \mathrm{ZnO}, \mathrm{ZnCoO}$, tungsten oxide, and $\mathrm{PbTiO}$ ), and finishing with $\mathrm{Bi}$ (a semimetal).

\title{
How to reference
}

In order to correctly reference this scholarly work, feel free to copy and paste the following:

Xue-Wen Chen and Sailing He (2010). Optical Modeling of Photoluminescence of Multilayered Semiconductor Nanostructures: Nanowires, Nanotubes and Nanocables, Nanowires, Paola Prete (Ed.), ISBN: 978-953-761979-4, InTech, Available from: http://www.intechopen.com/books/nanowires/optical-modeling-ofphotoluminescence-of-multilayered-semiconductor-nanostructures-nanowires-nanotub

\section{INTECH}

open science | open minds

\section{InTech Europe}

University Campus STeP Ri

Slavka Krautzeka 83/A

51000 Rijeka, Croatia

Phone: +385 (51) 770447

Fax: +385 (51) 686166

www.intechopen.com
InTech China

Unit 405, Office Block, Hotel Equatorial Shanghai

No.65, Yan An Road (West), Shanghai, 200040, China

中国上海市延安西路65号上海国际贵都大饭店办公楼405单元

Phone: +86-21-62489820

Fax: $+86-21-62489821$ 
(C) 2010 The Author(s). Licensee IntechOpen. This chapter is distributed under the terms of the Creative Commons Attribution-NonCommercialShareAlike-3.0 License, which permits use, distribution and reproduction for non-commercial purposes, provided the original is properly cited and derivative works building on this content are distributed under the same license. 